\title{
O SERVIÇO SOCIAL NO PROGRAMA DE TRANSPLANTE DE CORAÇÃO: AVALIAÇÃO SOCIAL
}

\section{Social Work in heart transplantation program: a social assessment}

\author{
Ana Lucia da Silva, Lucinei Paz Ferreira
}

\section{RESUMO}

Objetivo: Identificar os fatores sociais, econômicos, culturais e ambientais que influenciaram as condições de vida e saúde de candidatos ao programa de Transplante Cardíaco para subsidiar a equipe multiprofissional. Método: O estudo realizado foi quantitativo, descritivo e retrospectivo realizado no período de janeiro de 2006 a dezembro de 2009 no Instituto do Coração por meio de entrevista estruturada com amostragem de 526 pacientes e famílias. Resultado: O perfil dos pacientes caracterizou-se pela predominância do sexo masculino 67\%; com concentração de 34\% na faixa etária de 51 a 60 anos; $50 \%$ escolaridade correspondente ao ensino fundamental incompleto; $55 \%$ católicos; $67 \%$ com companheiro(a); 78\% apresentaram cuidadores, 54\% procedentes do Estado de São Paulo; $54 \%$ utilizaram transporte coletivo; $72 \%$ possuíam imóvel próprio; $92 \%$ dos domicílios apresentaram infra-estrutura e saneamento básico; $68 \%$ recebiam benefício previdenciário ou assistencial; 37\% com renda per capita de um a dois salários mínimos; 97\% com vinculação ao SUS. No total de avaliações sociais realizadas, 46\% apresentaram condições sociais adequadas ao acompanhamento do programa; 47\% permanecem em atendimento pelo Serviço Social para o monitoramento das condições sociais passiveis de alteração e 7\% dos pacientes apresentaram dificultadores que contra-indicaram a inclusão em lista. Conclusão: A avaliação social constitui ferramenta de valor prognóstico para mensuração das condições sociais de vida de cada indivíduo, para identificar os fatores socioeconômicos, culturais e ambientais presentes no cotidiano do paciente e suas famílias, que possam facilitar, interferir ou obstruir o processo de adesão, reintegração social e qualidade de vida.

Descritores: Transplante de Coração; Avaliação; Serviço Social.

\section{Instituição:}

Serviço Social Médico do Instituto do Coração (INCOR) - HCFMUSP São Paulo/SP, Brasil

\author{
Correspondência: \\ Ana Lucia da Silva \\ Av. Dr. Enéas de Carvalho Aguiar, 44 - CEP 05403-000 - São Paulo /SP \\ Telefone: (11) 2661-5381 \\ E-mail: silva.ana@incor.usp.br
}

Aceito em: 03.10.2011

\section{INTRODUÇÃO}

O principal ponto referente à saúde aprovado na Constituição de 1988 foi seu entendimento como direito universal e dever do Estado, sendo de competência do Sistema Único de Saúde (SUS) integrar todos os Serviços Públicos em uma rede hierarquizada, regionalizada, descentralizada e de atendimento integral com a participação da comunidade, garantindo-lhe acesso gratuito aos serviços para tratar e/ou recuperar a saúde.

As condições de saúde encontram-se estreitamente relacionadas, interligadas e influenciadas por diversos fatores, não sendo possível isolar o efeito de cada variável sobre os padrões de saúde da população. ${ }^{1}$

Segundo a Organização Mundial de Saúde (OMS), as doenças cardiovasculares (DCVS), denominadas crônicas representarão até o ano de $202060 \%$ de todos os óbitos do mundo. ${ }^{2}$

O desenvolvimento das DCVS pode resultar no surgimento de doença cardíaca com alto índice de mortalidade conhecida como insuficiência cardíaca (IC), síndrome que consiste na incapacidade de o coração liberar sangue para atender as necessidades metabólicas dos tecidos, a despeito de adequadas pressões de enchimento. $^{3}$

A cronicidade da doença cardíaca é caracterizada pelo tempo prolongado do seguimento médico, evidenciado pelas limitações da vida diária. Pode ocasionar alto nível de dependência de cuidados de terceiros, principalmente da família que na maioria das vezes é a principal provedora dos cuidados. 
O transplante cardíaco é a única forma eficaz de restaurar as funções hemodinâmicas do paciente; contudo apresenta limitações de diferentes ordens, não se aplicando a todos os candidatos. ${ }^{4}$

A indicação do transplante cardíaco surge como proposta não somente de salvar a vida dos pacientes, mas também oferecer melhora na sua qualidade de vida, na medida em que lhe possibilita retornar as atividades simples do cotidiano, com vistas à reinserção ao mundo do trabalho.

O prolongamento da vida é cada vez menos um desafio técnico para a ciência e cada vez mais é valorizada a qualidade de vida em detrimento do aumento do tempo que se vive em condições limitadas ou incapacitadas. ${ }^{5}$

Dessa forma, é exigido tanto do indivíduo doente quanto dos seus familiares e cuidadores novos arranjos de enfrentamento para as alterações cotidianas decorrentes da doença, com repercussões nas relações sociais.

O programa de transplante de coração do Incor apresenta índices de sobrevida dos pacientes selecionados para operação equivalente aos internacionais e vem se consolidando num dos principais centros transplantadores de órgãos da América Latina. Conta com suporte de equipe multiprofissional especializada na seleção dos receptores.

O Assistente Social no programa realiza avaliação social com os candidatos e familiares que vai além do apontamento à equipe médica e multiprofissional de questões sociais que indiquem ou contra indiquem a realização do transplante, mas que possibilita estabelecer plano de intervenção às questões com poder de comprometer a adesão ao tratamento.

A delimitação do objetivo deste estudo configurou-se em identificar os fatores sociais, econômicos, culturais e ambientais que influenciaram as condições de vida e de saúde dos candidatos, em relação ao processo de adesão, reintegração social e qualidade de vida, necessários a uma adequada efetivação do programa de transplante cardíaco. Compreendemos que tal avaliação pode qualificar ainda mais o trabalho de toda a equipe multiprofissional.

\section{MÉTODO}

O estudo utilizou o método quantitativo, retrospectivo e descritivo. A amostra foi constituída de 526 avaliações sociais realizadas com pacientes e familiares com indicação para transplante cardíaco, no Instituto do Coração, no período de janeiro de 2006 a dezembro de 2009.

A coleta de dados foi recuperada dos relatórios sociais, que são produtos das entrevistas individuais ou em grupo familiar subdivididos em perfil sócio demográfico contendo variáveis (sexo, idade, estado conjugal, religião, escolaridade, procedência, transporte, vínculo previdenciário, condições de habitação, renda familiar) e critérios de elegibilidade, compostos por indicadores que definem o quadro social de cada paciente e que possibilitou identificar os aspectos facilitadores e/ou dificultadores da adesão do paciente ao programa de transplante. As variáveis contidas neste bloco de analise foram:

- Aceitação: Dificuldade do paciente em aceitar a doença e o transplante (negação). A presença de valores e crenças individuais ou familiares que comprometam o tratamento e/ou abandono do mesmo;

- Acesso: Dificuldade de acesso ao hospital, entendido como capacidade do paciente comparecer ao Centro Transplantador no prazo de tempo recomendado (duas horas) por ocasião do transplante e comparecimento nas consultas no pós-transplante cardíaco.

- Condições sócio-econômicas: Habitação com infra-estrutura parcial, ausência de saneamento básico, imóveis sem redes de esgotos ou insalubres. Renda familiar insuficiente para garantia das necessidades básicas (renda per capita inferior a meio salário mínimo). ${ }^{6}$

- Dinâmica familiar: Ausência de familiares e ou colateral para assumir o papel de cuidador, ou seja, com capacidade de tomar decisões voltadas para o apoio ao paciente nas diferentes atividades de sua vida diária, como também no uso adequado de medicamentos, colaborando em todas as fases do tratamento.

\section{RESULTADOS}

Os indicadores sócio-demográficos e culturais presentes na pesquisa com 526 pacientes caracterizaram-se pela predominância do sexo masculino $67 \%$, com concentração de $34 \%$ na faixa etária de 51 a 60 anos, seguidos de $25 \%$ na faixa etária de 41 a 50 anos.

A escolaridade dos pacientes caracterizou-se por $50 \%$ correspondente ao ensino fundamental incompleto, seguido por 19\% com ensino médio completo.

A maioria dos pacientes (67\%) referiu estado conjugal com companheira, declarando-se 55\% católicos, seguidos de $23 \%$ evangélicos.

$\mathrm{Na}$ amostra, $45 \%$ dos cuidados com pacientes são desempenhados pelas companheiras e $22 \%$ da população estudada não apresentaram cuidador.

A população estudada foi caracterizada pela predominância de brasileiros (100\%). Destes, 97\% vinculados ao Sistema Único de Saúde (SUS) e apenas 3\% beneficiários de convênios médicos; $54 \%$ naturais e procedentes do Estado de São Paulo, com igualdade de $20 \%$ de pacientes da região sudeste e nordeste.

O acesso caracterizou-se como: 54\% utilizaram transporte coletivo, $27 \%$, transporte próprio ou de terceiros e 19, transporte cedido pelo Estado ou Município via Tratamento Fora de Domicilio (TFD).

A variável habitação demonstrou: $72 \%$ da população residiam em imóvel próprio, 16\% em alugado e 12\% em imóvel cedido.

Com relação ao saneamento básico e adequação de material de construção, 92\% dos domicílios apresentaram-se satisfatórios, 7\% parcial e $1 \%$ inexistente.

Ao analisarmos os pacientes afastados do setor produtivo, verificamos que $36 \%$ dos pacientes encontravam-se aposentados, $28 \%$ em auxílio doença e 4\% em gozo de benefício assistencial; os demais $32 \%$ não recebiam qualquer beneficio, uma vez que não possuíam cobertura da legislação trabalhista.

A variável renda per capita apresentou-se: 37\% recebiam entre um a dois salários mínimos, seguidos de $34 \%$ com renda de dois a cinco salários mínimos.

Nas avaliações sociais realizadas com os pacientes, $46 \%$ apresentaram condições sociais facilitadoras ao adequado acompanhamento do programa, $47 \%$ permaneceram em atendimento pelo Serviço Social para se monitorarem as condições sociais e familiares passíveis de alteração e 7\% apresentaram 
dificultadores que contra indicaram a inclusão em lista única de receptores gerenciada pela Secretária de Saúde do Estado de São Paulo.

\section{DISCUSSÃO}

O exercício do Serviço Social tem por horizonte os direitos sociais. Esses foram garantidos pela Seguridade Social quando da elaboração da atual Constituição brasileira, que afirma em seu artigo 194; "A seguridade social compreende um conjunto integrado de ações de iniciativa dos Poderes Públicos da sociedade, destinadas a assegurar os direitos relativos à saúde, a previdência e à assistência social"?

O SUAS Sistema Único de Assistência Social, cujo modelo de gestão é descentralizado e participativo, constitui-se na regulação e organização em todo o território nacional das ações sócioassistenciais. Os serviços, programas, projetos e benefícios têm como foco prioritário a atenção às famílias, seus membros e indivíduos e o território como base de organização, que passam a ser definidos pelas funções que desempenham, pelo número de pessoas que deles necessitam e pela sua complexidade, visando consagrar direitos de cidadania e inclusão social. ${ }^{8}$

As múltiplas manifestações e representações que o processo de doença ocasionaram no contexto sócio familiar dos candidatos a receptores do coração, aliadas às questões sociais de exclusão ao programa, exigiram do Assistente Social estratégias de ação na busca de soluções eficazes à efetivação de direitos sociais, de inclusão, sem perder de vista também as demandas que necessitaram de intervenções imediatas, mesmo que paliativas, com vistas à materialização do suprimento das carências socioeconômicas dos candidatos, tais como:

- encaminhamento para programas sociais que oferecessem, auxilio para transporte, doação de cesta básica alimentar, etc.

- entrosamento com organizações sociais $\left(\mathrm{ONG}^{\prime} \mathrm{S}\right)$ para suporte quanto ao alojamento daqueles procedentes de outros Estados;
- captação de recursos internos para auxílio às questões relacionadas ao tratamento, entre outras.

$\mathrm{O}$ caráter emancipatório do atendimento social ocorreu quando se viabilizou a inclusão dos candidatos aos programas socioassistenciais garantidos pelo SUAS, como por exemplo: CRAS (Centro de Referência da Assistência Social), Prefeitura, Secretária de Saúde, Secretária de Habitação, etc. que ofereceram capacitação na superação da situação social apresentada,(renda, habitação, previdência social, transporte etc.) e na capacidade denotada pelo candidato no empenho em transformar possibilidades em efetividades, uma vez que, independente do contexto social, deveria ser responsável pela construção da sua história, para que de fato a sua inclusão em fila de espera pelo órgão fosse efetivada.

\section{CONCLUSÃO}

O momento da avaliação social constituiu-se, muitas vezes, em situação de angústia para o Assistente Social, ao deparar-se com os entraves sociais dos candidatos que à primeira vista, pareciam intransponíveis pelo desconhecimento demonstrado por parcela deles quanto aos direitos sociais garantidos pelas políticas públicas, que na teoria prevêm a igualdade de contextos sociais tão desiguais.

À medida, porém, em que os atendimentos sociais eram realizados, as visitas domiciliares efetuadas e os serviços socioassistenciais promoviam a inclusão dos receptores junto aos serviços solicitados, deparamo-nos com um Ser-Sujeito agente e modificador da sua condição de iniquidade social, amparado pelo poder público, garantindo a sua inclusão no programa de transplante.

Frente às situações sociais apresentadas pelos nossos pacientes, faz-se necessário pensar na precariedade do Atendimento Básico de Saúde do país e no impacto que ações preventivas de saúde poderão desencadear junto aos nossos pacientes e à comunidade, no enfrentamento das vulnerabilidades sociais, sanitárias e ambientais, contribuindo até mesmo com a diminuição da indicação para transplante de órgãos.

\section{ABSTRACT:}

Purpose: The aim of this study was to identify social, economic, cultural and environmental conditions that influenced the life and health of candidates for the transplantation program to provide subsidies to the multidisciplinary team. Methods: It was a quantitative, descriptive and retrospective study performed from January 2006 to December 2009 at the Heart Institute through structured interviews with sampling of 526 patients and their families. Results: The profile of patients was characterized by $67 \%$ of male predominance with $34 \%$ concentration of 51 to 60 years old patients; as to the school level, $50 \%$ corresponded to elementary school, $55 \%$ catholic; as to the marital status: $67 \%$ had spouse, $78 \%$ had caretakers, 54\% were originated in the State of São Paulo, 54\% used public transportation, $72 \%$ had their own home, $92 \%$ of domiciles had infrastructure and basic sanitation, $68 \%$ received social security benefits or assistance, $37 \%$ had per capita income of 1 to 2 minimum wages, $97 \%$ were linked to SUS. From the total social assessments carried out, $46 \%$ had social conditions for the proper monitoring of the program, $47 \%$ remained assisted by Social Workers to monitor social and family conditions subject to change, and $7 \%$ of patients presented hindering against their inclusion on the list. Conclusion: The social assessment is a prognostic tool to measure social life conditions of each individual, in order to identify socioeconomic and cultural factors in the daily routine of patients and their families that can facilitate, interfere or obstruct in their adhesion process, social reintegration and quality of life.

Keywords: Heart transplant; Evaluation; Social Service. 


\section{REFERÊNCIAS:}

1. Sareta FO. Serviço Social: A prática profissional na Saúde Pública. ANAIS da III Semana de Serviço Social; 2004. p. 339-51.

2. Brasília, Ministério da Saúde. Cuidados inovadores para condições crônicas: componentes estruturas de ações. Relatório mundial. Brasília.OMS; 2003. Guimarães IRF. Dimensão educacional do amor. In: As dimensões do amor Campinas: Unicamp;1993.p.173.

3. Mesquita ET. Avaliação clinica e diagnóstico de insuficiência cardíaca. In: Barreto, ACP; Bocchi, EA (orgs): Insuficiência cardíaca. São Paulo: Segmento; 2003. p 41.

4. Fiorelli AI, Lima Jr JL, Stolf AGN. Transplante Cardíaco. Rev. Méd (São Paulo); 2009. p.123-37.
5. Moacyr RCN. Qualidade de Vida [editorial]. Arq Bras Cardiol. 1995;64(4):299-300.

6. Lemos C, Neves N. Métodos de avaliação social em programa de transplante cardíaco. Rev Soc. Cardiol Estado de São Paulo. 2000; 10 (1 supl. A): 11-20.

7. Silva RCP, Arizono AD. A política nacional de humanização do SUS e o Serviço Social. Revista Ciências Humanas. São Paulo, volume 1, $\mathrm{n}^{\circ} 2$; ano 2008;1-10.

8. Brasil, Sistema Único de Assistência Social/SUAS, Lei n. 12.435, de 6 de julho de 2011. 